\title{
Identification of Two Distinct Progenitor Populations in the Lateral Ganglionic Eminence: Implications for Striatal and Olfactory Bulb Neurogenesis
}

\author{
Jan Stenman, ${ }^{1,2}$ Håkan Toresson, ${ }^{2}$ and Kenneth Campbell ${ }^{1}$ \\ ${ }^{1}$ Division of Developmental Biology, Children's Hospital Research Foundation, Cincinnati, Ohio 45229-3039, and ${ }^{2}$ Wallenberg Neuroscience Center, \\ Division of Neurobiology, Lund University, S-221 84 Lund, Sweden
}

The lateral ganglionic eminence (LGE) is known to give rise to striatal projection neurons as well as interneurons, which migrate in the rostral migratory stream (RMS) to populate the granule cell and glomerular layers of the olfactory bulb. Because all of these neuronal subtypes express Distalless-related (DLX) homeobox proteins during their differentiation, we set out to further characterize progenitors in the Dlx-positive domain of the LGE. Previous studies have shown that the LIM homeobox protein Islet1 (ISL1) marks the LGE subventricular zone (SVZ) and differentiating striatal projection neurons. However, ISL1 is not expressed in neurons of the developing olfactory bulb or the RMS. We show here that the dorsal-most portion of the Dlx-expressing region of the LGE SVZ lacks ISL1 cells. This dorsal domain, however, contains cells that express the ETS transcription factor Er81, which is also expressed in granule and periglomerular cells of the developing and adult olfactory bulb. Moreover, the adult SVZ and RMS contain numerous Er81-positive cells. Fate-mapping studies using Dlx5/6-cre transgenic mice demonstrate that Er81-positive cells in the granule cell and glomerular layers of the olfactory bulb derive from the $D l x$-expressing SVZ region. These findings suggest that the LGE SVZ contains two distinct progenitor populations: a $\mathrm{DLX}^{+}{ } \mathrm{ISL1}^{+}$population representing striatal progenitors and a $\mathrm{DLX}^{+}{ }^{+}$Er81 ${ }^{+}$population comprising olfactory bulb interneuron progenitors. In support of this, mice mutant for the homeobox genes $G \operatorname{sh} 2$ and $G \operatorname{sh} 1 / 2$, which show olfactory bulb defects, exhibit dramatically reduced numbers of Er81-positive cells in the LGE SVZ as well as in the olfactory bulb mantle.

Key words: DLX; Er81; Gsh1; Gsh2; Islet1; NKX2.1; Small eye; telencephalon

\section{Introduction}

The extensive neuronal diversity of the mature nervous system is central to its many complex functions. Recent studies have begun to uncover some of the mechanisms that regulate the generation of this diversity during nervous system development. At spinal cord levels, distinct neuronal subtypes have been shown to arise from discrete progenitor domains positioned along the dorsoventral axis (Jessell, 2000). Combinatorial codes of transcription factors define these progenitor domains and appear to regulate the development of specific neuronal populations, such as motor neurons versus interneurons (Briscoe et al., 2000). Similar progenitor domains have not, as yet, been well defined in the anterior regions of the developing brain.

Recent studies have shown that both radial and tangential migration contributes significantly to neuronal diversity within distinct telencephalic regions (for review, see Marin and Rubenstein, 2001). Whereas projection neurons seem to be generated from the germinal zones directly adjacent to the telencephalic structure they ultimately populate, interneurons appear to be produced in restricted regions and undergo extensive migration to reside in different telencephalic regions. An example of this

\footnotetext{
Received June 13, 2002; revised Sept. 17, 2002; accepted Sept. 25, 2002.

This work was supported by funds from the Children's Hospital Research Foundation and the Human Frontiers Science Program (RG160-2000B). We thank M. Ekker for providing the id6/id5 element, S. Morton and T. Jessell for the Er81 antibody, and G. Panganiban for the Distalless (DLX) antibody.

Correspondence should be addressed to Kenneth Campbell at the above address. E-mail: kenneth.campbell@chmcc.org.

Copyright $\odot 2002$ Society for Neuroscience $\quad 0270-6474 / 02 / 220167-08 \$ 15.00 / 0$
}

occurs in the medial ganglionic eminence (MGE), where a number of distinct interneuronal populations are generated and subsequently migrate tangentially to populate distant telencephalic regions, such as the striatum, cortex, and hippocampus (Lavdas et al., 1999; Pleasure et al., 2000; Wichterle et al., 1999, 2001; Anderson et al., 2001). Another example is in the lateral ganglionic eminence (LGE), which has been shown to give rise to striatal projection neurons (Deacon et al., 1994; Olsson et al., 1995, 1997, 1998; Wichterle et al., 2001) as well as interneurons that migrate in the rostral migratory stream (RMS) and populate both the glomerular and granule cell layers of the olfactory bulb (Wichterle et al., 1999, 2001). That both striatal projection neurons and olfactory bulb interneurons are derived from the LGE is consistent with data from Mash1 and Dlx1/2 homozygous mutants (Anderson et al., 1997; Bulfone et al., 1998; Casarosa et al., 1999). Both of these genes are expressed in cells of the ventricular zone (VZ) and/or subventricular zone (SVZ) of the LGE, and the loss-of-function mutants show differentiation defects in both the striatum and the olfactory bulb. Moreover, in Gsh2 mutants, which have reduced Mash1 and Dlx gene expression in the LGE, leaving their expression in the MGE relatively unaffected, both striatal and olfactory bulb defects are observed (Corbin et al., 2000; Toresson et al., 2000; Toresson and Campbell, 2001; Yun et al., 2001). Distinct subtypes of cortical and hippocampal interneurons have also been suggested to arise from the LGE at later stages of neurogenesis (Pleasure et al., 2000; Anderson et al., 2001).

It remains unclear whether all of these LGE-derived neuronal subtypes are generated from a common progenitor or whether 
distinct progenitor pools exist in the LGE, as is the case in the spinal cord. We provide evidence here that at least two distinct progenitor pools exist in the LGE, and suggest that they contribute differentially to striatal and olfactory bulb neurogenesis.

\section{Materials and Methods}

Animals: Generation of Dlx5/6-cre-IRES-EGFP mice. cre recombinase cDNA was subcloned into the pIRES2EGFP vector (Clontech, Palo Alto, CA). cre-internal ribosome entry site (IRES)-enhanced green fluorescent protein $(E G F P)$ was subsequently subcloned into a vector containing the mouse id6/id5 enhancer (Zerucha et al., 2000) provided by Dr. M. Ekker (University of Ottawa, Ottawa, Canada). Pronuclear injections of the Dlx5/6-cre-IRES-EGFP construct were performed by the transgenic core at Children's Hospital Research Foundation. Founders were identified by PCR using the following primers: GFP5, CTA ACG TTA CTG GCC GAA; GFP3, ACT TGA AGA AGT CGT GCT. Three independent lines were established, and all show similar expression of the transgene. The Dlx5/ 6-cre-IRES-EGFP mice were kept on a C57BL/6 background. Heterozygote embryos and postnatal day $0(\mathrm{P} 0)$ pups were obtained from heterozygote and wild-type crosses and typed by visual inspection for enhanced green fluorescent protein (EGFP) under a fluorescent microscope. Adult Dlx5/6-cre-IRES-EGFP/gtROSA double transgenic brains were obtained by crossing heterozygous B6;129-Gtrosa26 ${ }^{\text {tm1Sho }}$ ( $g t R O S A$ ) mice (Mao et al., 1999) (obtained from The Jackson Laboratory, Bar Harbor, ME) with hemizygous Dlx5/6-cre-IRES-EGFP mice.

Gsh1 (Li et al., 1996), Gsh2 (Szucsik et al., 1997), and Small eye (Sey) mice and embryos were typed as described previously (Toresson et al., 2000; Toresson and Campbell, 2001). gtROSA mice were genotyped as described in the Gtrosa26 genotyping protocol of The Jackson Laboratory (http://www.jax.org). For staging of embryos, the morning of vaginal plug was designated as embryonic day 0.5 (E0.5).

Immunohistochemistry. Embryos and P0 pups were processed as described previously (Toresson et al., 2000). Adult brains were removed fresh, immersion fixed in $4 \%$ paraformaldehyde in PBS overnight at $4^{\circ} \mathrm{C}$, and then placed in PBS with $30 \%$ sucrose for at least $72 \mathrm{hr}$ at $4^{\circ} \mathrm{C}$ before sectioning on a cryostat. The adult brains were either sectioned at $30-40$ $\mu \mathrm{m}$ and kept as free-floating in PBS or sectioned at $8 \mu \mathrm{m}$ and mounted directly onto slides. Immunohistochemistry was performed on the slidemounted sections as described previously (Olsson et al., 1997), with the modification that only $0.3 \% \mathrm{H}_{2} \mathrm{O}_{2}$ was used for $10-15$ min instead of $3 \%$ $\mathrm{H}_{2} \mathrm{O}_{2}$. The 30- to $40-\mu \mathrm{m}$-thick adult brain sections were immunostained free-floating and subsequently mounted onto slides. Primary antibodies were used at the following concentrations: rabbit anti-Distalless (i.e., DLX) (1:1000; provided by G. Panganiban, University of WisconsinMadison, Madison, WI), rabbit anti-Er81 (1:5000; provided by S. Morton and T. Jessell, Columbia University, New York, NY), chicken antiGFP (1:5000; Chemicon, Temecula, CA), rabbit anti-Islet1/2 (ISL1/2) (1:500; provided by T. Edlund, Umeå University, Umeå, Sweden), and goat anti-NKX2.1 (1:1200, Santa Cruz Biotechnology, Santa Cruz, CA). For fluorescent staining, donkey anti-rabbit antibodies conjugated to Cy3 (Jackson ImmunoResearch, West Grove, PA) or donkey antichicken antibodies conjugated to FITC (Jackson ImmunoResearch) were used as secondary antibodies (Jackson ImmunoResearch). NKX2.1 was visualized using biotinylated horse anti-goat antibodies (Vector Laboratories, Burlingame, CA) and streptavidin conjugated with FITC for fluorescent detection (Jackson ImmunoResearch). Confocal microscopy was performed using a Zeiss (Thornwood, NY) LSM510 confocal microscope. For bright-field staining, biotinylated swine anti-rabbit antibodies (Dako, Carpinteria, CA) were used with the ABC kit (Vector Laboratories) and diaminobenzidine (Sigma, St. Louis, MO) as the final chromogen.

5-bromo-4-chloro-3-indolyl- $\beta$-D-galactopyranoside histochemistry. 5bro-mo-4-chloro-3-indolyl- $\beta$-D-galactopyranoside (X-gal) staining was performed on both slide-mounted and free-floating sections. First, they were washed in $0.02 \% \mathrm{NP}-40$ and $2 \mathrm{mM} \mathrm{MgCl}_{2}$ in PBS. They were then incubated in the staining solution $\left(5 \mathrm{~mm} \mathrm{~K}_{3} \mathrm{Fe}(\mathrm{CN})_{6}, 5 \mathrm{~mm} \mathrm{~K}_{4} \mathrm{Fe}(\mathrm{CN})_{6}, 2\right.$ $\mathrm{mm} \mathrm{MgCl} 2,0.01 \%$ deoxycholate, $0.02 \% \mathrm{NP}-40$, and $1 \mathrm{mg} / \mathrm{ml} \mathrm{X-gal)} \mathrm{at}$ $37^{\circ} \mathrm{C}$ until the stain was clearly visible. The sections or slides were then transferred to a potassium PBS solution and processed for immunohistochemistry as described above.

\section{Results}

\section{ISL1 overlaps extensively with the Dlx5/6-expressing domain of the LGE}

The LGE is known to give rise to striatal projection neurons as well as interneurons populating the olfactory bulb (Deacon et al., 1994; Olsson et al., 1997, 1998; Wichterle et al., 2001). However, it is not clear whether these neurons are generated from a common progenitor cell in the SVZ of the LGE or from distinct progenitor pools. Because both striatal projection neurons and olfactory bulb interneurons express DLX proteins during their differentiation, we set out to further characterize progenitors in the $D l x$-positive domain of the LGE.

To aid in this analysis, we made transgenic mice expressing cre recombinase and EGFP from a bicistronic construct under the control of the Dlx5/6 enhancer element id6/id5 (Zerucha et al., 2000). At E12.5, DLX proteins, including DLX1, DLX2, DLX5, and DLX6 (recognized by an antibody against Drosophila Distalless) (Panganiban et al., 1995) are expressed throughout the MGE and the LGE, including both the VZ and the SVZ (Fig. $1 A$ ). EGFP expression from the $D l \times 5 / 6$ enhancer is confined to the SVZ and forming mantle (Fig. $1 \mathrm{~B}$ ), and merged confocal images show that EGFP-expressing cells constitute a subdomain of the DLXpositive domain (Fig. 1C). This is in accordance with the expression of lac $Z$ from the Dlx5/6 enhancer (Zerucha et al., 2000; Stuhmer et al., 2002) as well as the expression of the endogenous Dlx5 and Dlx6 genes (Liu et al., 1997). At E16.5, DLX proteins are again found in the VZ, SVZ, and mantle regions (Fig. $2 A$ ). However, the Dlx5/6-driven EGFP is confined to the SVZ and mantle (Fig. $2 B, C$ ), closely resembling the endogenous $D l \times 5 / 6$ expression pattern.

Previous studies have demonstrated that the LIM homeobox protein ISL1 is expressed in differentiating striatal neurons, including the projection neurons and cholinergic interneurons (Toresson et al., 2000; Toresson and Campbell, 2001; Wang and Liu, 2001). The ISL1-expressing cells in the SVZ of the LGE (Figs. $1 D, 2 D)$ and in the developing striatum (Fig. $2 D$ ) overlap extensively with the domain expressing EGFP from the Dlx5/6 enhancer (Figs. $1 F, 2 F$ ). However, in the dorsal-most portion of the LGE, an EGFP-positive (i.e., $\mathrm{DLX}^{+}$) and ISL1-negative domain is evident both at E12.5 (Fig. $1 F$ ) and at E16.5 (Fig. 2F). Thus already at E12.5, two separate SVZ progenitor pools can be identified in the Dlx5/6-expressing domain of the LGE: one defined by the expression of ISL1 $\left(\mathrm{DLX}^{+}{ }^{+} \mathrm{ISL}^{+}{ }^{+}\right.$) and another by its absence $\left(\mathrm{DLX}^{+} ; \mathrm{ISL}^{-}{ }^{-}\right)$. The overlap in the SVZ expression of $\mathrm{Dl} \times 5 / 6-$ driven EGFP and ISL1 is greatly reduced at P0 (Fig. $3 A$ ). This is in accordance with the time course of striatal neurogenesis, which ends around birth (Bayer and Altman, 1995). As mentioned above, olfactory bulb interneurons in both the granule cell and periglomerular layers are also derived from the Dlx5/6-expressing LGE SVZ (Fig. 3B); however, at no stage examined have we observed ISL1-positive olfactory bulb neurons (Fig. 3B) (data not shown). Thus the $\mathrm{DLX}^{+} ; \mathrm{ISL}^{+}{ }^{+}$domain in the LGE SVZ may contain progenitors that are restricted to striatal neuron fates.

\section{Er81 marks a subdomain of the Dlx5/6-expressing LGE}

To further characterize the $\mathrm{DLX}^{+}{ } \mathrm{ISL}^{-}{ }^{-}$domain of the LGE SVZ, we looked for markers expressed in this region. A recent study by Yun et al. (2001) demonstrated that the ETS transcription factor gene Er81 is expressed in the dorsal-most portion of the LGE VZ. Although the protein expression of Er81 is not de- 

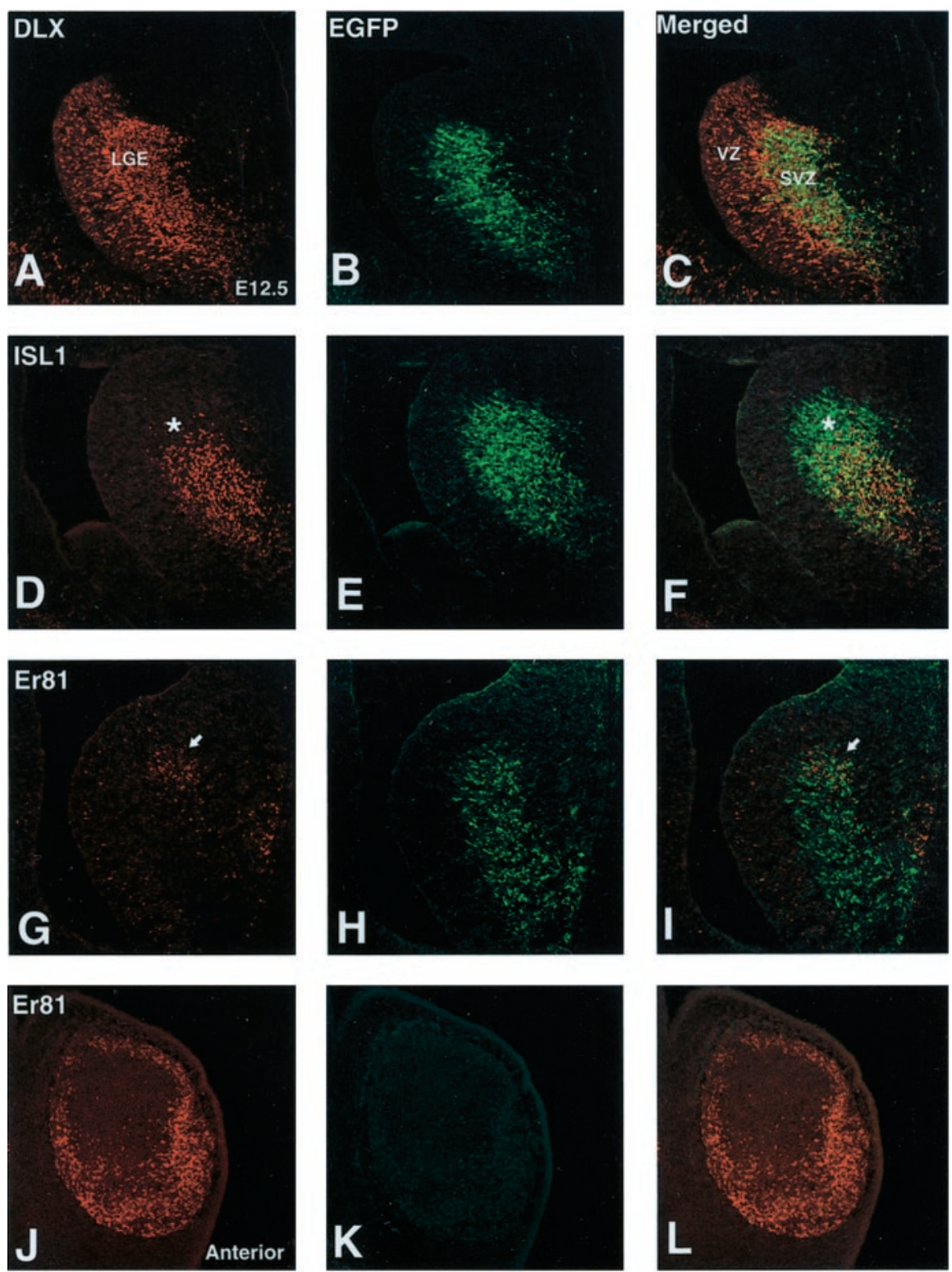

Figure 1. Expression of $\operatorname{DLX}(A), \operatorname{ISL1}(D)$, Er81 $(G, J)$, and EGFP expressed from the $D / x 5 / 6$ element $(B, E, H)$ atE $12.5 . A, D L X$ proteins are expressed in cells of the VZ and SVZ of the LGE, whereas the EGFP-expressing cells are predominantly localized to the SVZ (B, O.D, ISL1 expression is found in the SVZ and overlaps extensively with EGFP expression $(E, F)$. Note, however, the lack of ISL1 in the dorsal-most portion of the EGFP-expressing domain (asterisk in Dand $F$ ). In the dorsal portion of the LGE, Er81-positive cells ( $G$ ) overlap with the dorsal $D / x 5 / 6$-driven EGFP expression $(H, I)$.The arrowin Gand/points to the Er81-positive cell cluster in the DLX domain. Atmore anterior regions, Er81 expression is seen in the olfactory bulb primordium (J). Note that at this stage EGFP (i.e., D/x5/6) expression is not detected at the anterior level $(K, L)$.

tected at high levels in the LGE VZ, we observed that at both E12.5 (Fig. 1G-I) and E16.5 (Fig. 2G-I), Er81 is expressed in the DLX ${ }^{+}$; ISL1 ${ }^{-}$domain of the SVZ, which presumably lies directly under the region of the LGE VZ expressing the Er81 gene (Yun et al., 2001). This expression domain is most evident at rostral levels of the LGE. After birth (i.e., P0), many cells in the postnatal SVZ still coexpress EGFP from the Dlx5/6 enhancer and Er81 (Fig. 3C).

The expression of Er81 is by no means specific to the DLX ${ }^{+}$; ISL1 $^{-}$SVZ domain. It is also expressed in the VZ of the ventrolateral pallium (i.e., cortical VZ) (Fig. 2G), in the medial portion of the MGE, in the differentiating pallidum (including the globus pallidus) (Fig. $2 J$ ), as well as in scattered cells of the developing striatum (Fig. 2G). The scattered expression of Er81 in the developing striatum suggests that this transcription factor may mark striatal interneurons or a subpopulation thereof. Although it is possible that these cells could originate from the $\mathrm{DLX}^{+}$; Er8 $1^{+}$domain of the LGE SVZ, it is more likely that they originate from the adjacent MGE. Indeed, most striatal interneurons have been shown to derive from the MGE and subsequently migrate laterally into the developing striatum (Olsson et al., 1998; Sussell et al., 1999; Marin et al., 2000). Interneurons in the striatum are known to express the MGE-specific homeobox transcription factor NKX2.1 (Sussell et al., 1999; Marin et al., 2000). Indeed, the majority of Er81-expressing cells in the striatum also express NKX2.1 (Fig. $2 L$ ), indicating that they are likely derived from the MGE and represent striatal interneurons. Moreover, a stream of Er81/NKX2.1-coexpressing cells can be seen oriented in the direction of the striatum (Fig. $2 J, K$ ), suggestive of the migratory path these cells may take.

In addition to the $\mathrm{DLX}^{+} ; \mathrm{Er} 81^{+}$region in the LGE SVZ, Er81 is also found in the rostrally located DLX ${ }^{-}$primordia of the olfactory bulb at E12.5 (Fig. $1 J-L$ ). Expression of Er81 is maintained in the germinal zone of the olfactory bulb at later stages as well as in cells of the mantle region (Fig. 3D). Interestingly, EGFP driven from the $D l \times 5 / 6$ enhancer is found in cells of the developing olfactory bulb by E16.5, both in the germinal zone as well as in the developing granule and periglomerular cells (data not shown). Many of the EGFP-expressing cells in the P0 olfactory bulb were found to coexpress Er81, especially in the developing glomerular layer (Fig. 3D,E). These findings suggest that cells in the $\mathrm{DLX}^{+} ; \mathrm{Er} 81^{+}$region of the LGE SVZ could represent olfactory bulb interneuron progenitors.

\section{Er81 in the adult SVZ and \\ olfactory bulb}

Neurogenesis in the olfactory bulb is known to continue throughout the postnatal period and into adulthood (Luskin, 1993; Lois and Alvarez-Buylla, 1994; Bayer and Altman, 1995). At present, the relationship between the LGE and the postnatal/ adult SVZ is not clear; however, the postnatal/adult SVZ comes to reside on the ventricular wall of the striatum (the principal derivative of the LGE). Moreover, cells in the postnatal/adult SVZ express DLX proteins (Fig. 4A), suggestive of an LGE origin. Er81 is also detected in cells of the postnatal/adult SVZ (Fig. $4 B$ ). Furthermore, Er81 can be detected in cells of the RMS and in olfactory bulb neurons, in the granule cell layer, and in the glomerular layer (Fig. 4C,D). In the granule cell layer, Er81 expression is strongest in the outer regions and is also found in the granule cells of the mitral layer (Fig. 4D).

As suggested by the embryonic analysis, the $\mathrm{DLX}^{+} ; \mathrm{Er} 81^{+}$region may give rise to olfactory bulb interneurons. To address this 
issue, we crossed Dlx5/6-cre-IRES-EGFP mice with $g t R O S A$ reporter mice (Mao et al., 1999), which have a floxed "stop transcription" sequence in front of the lac $Z$ gene. In cells in which cre recombinase is expressed, the "stop" sequence is recombined out and the lac $Z$ is expressed. In this case, all cells that express or expressed the Dlx5/6 enhancer at some point will express $\beta$-galactosidase from the lac $Z$ gene. The striatum, which is known to be derived from the LGE (Olsson et al., 1997; Wichterle et al., 2001), shows many $\mathrm{X}$-gal-positive cells (Fig. 5A). Because the $\mathrm{X}$-gal reaction product is only a small dot located in the cytoplasm of the positive cell, we used thin (i.e., $8 \mu \mathrm{m}$ ) sections to colocalize the X-gal in subtypes of striatal and olfactory bulb neurons. In thin sections, the overwhelming majority of striatal neurons expressing dopamine and cAMP-regulated phosphoprotein (DARPP)-32, a marker of striatal projection neurons (Anderson and Reiner, 1991), coexpressed the X-gal reaction product (Fig. $5 B$ ). In addition to the $\mathrm{X}$-gal staining of striatal neurons, many cells were X-gal-positive in the granule cell and glomerular layers of the olfactory bulb (Fig. 5C). Analysis of thin sections showed that the majority of Er81-positive cells in the glomerular (Fig. 5D) and granule cell layers double-stain for X-gal (Fig. 5E). Moreover, the granule cells of the mitral layer coexpress Er81 and X-gal. These findings support the notion that periglomerular and at least a portion of the granule cells originate from the $\mathrm{DLX}^{+}$; Er81 ${ }^{+}$ LGE domain.

\section{Genetic regulation of the $\mathrm{DLX}^{+} ; \mathrm{Er} 81^{+}$ LGE domain}

Recent studies (Corbin et al., 2000; Stoykova et al., 2000; Toresson et al., 2000; Yun et al., 2001) have demonstrated that the developmental regulators $\operatorname{Pax} 6$ and Gsh2 are required for the maintenance of dorsoventral identity and, in particular, for the correct formation of the boundary between the pallium (i.e., developing cortex) and the LGE. In Gsh 2 homozygotes, the expression of Mash 1 and $D l x$ genes is lost from most of the progenitors in the LGE; in their place, the dorsal regulators Pax6, Neurogenin1 (Ngn1) and Ngn2 are expanded ventrally. The converse is the case in the Sey homozygotes (i.e., Pax6 mutants). We were interested in examining the $\mathrm{DLX}^{+} ; \mathrm{Er} 81^{+}$domain of the LGE SVZ in Gsh 2 and Pax6 mutants. In the Gsh2 mutant at E18.5, the ISL1 domain is decreased in size, as described previously (Toresson et al., 2000; Toresson and Campbell, 2001). Interestingly however, ISL1-positive cells occupy the dorsal-most portion of the mutant LGE (Fig. $6 B$ ), unlike in the wild type (Fig. $6 A$ ). The reduction in ISL-positive cells in the LGE of Gsh1/2 double homozygous mutants is even more severe than that in the
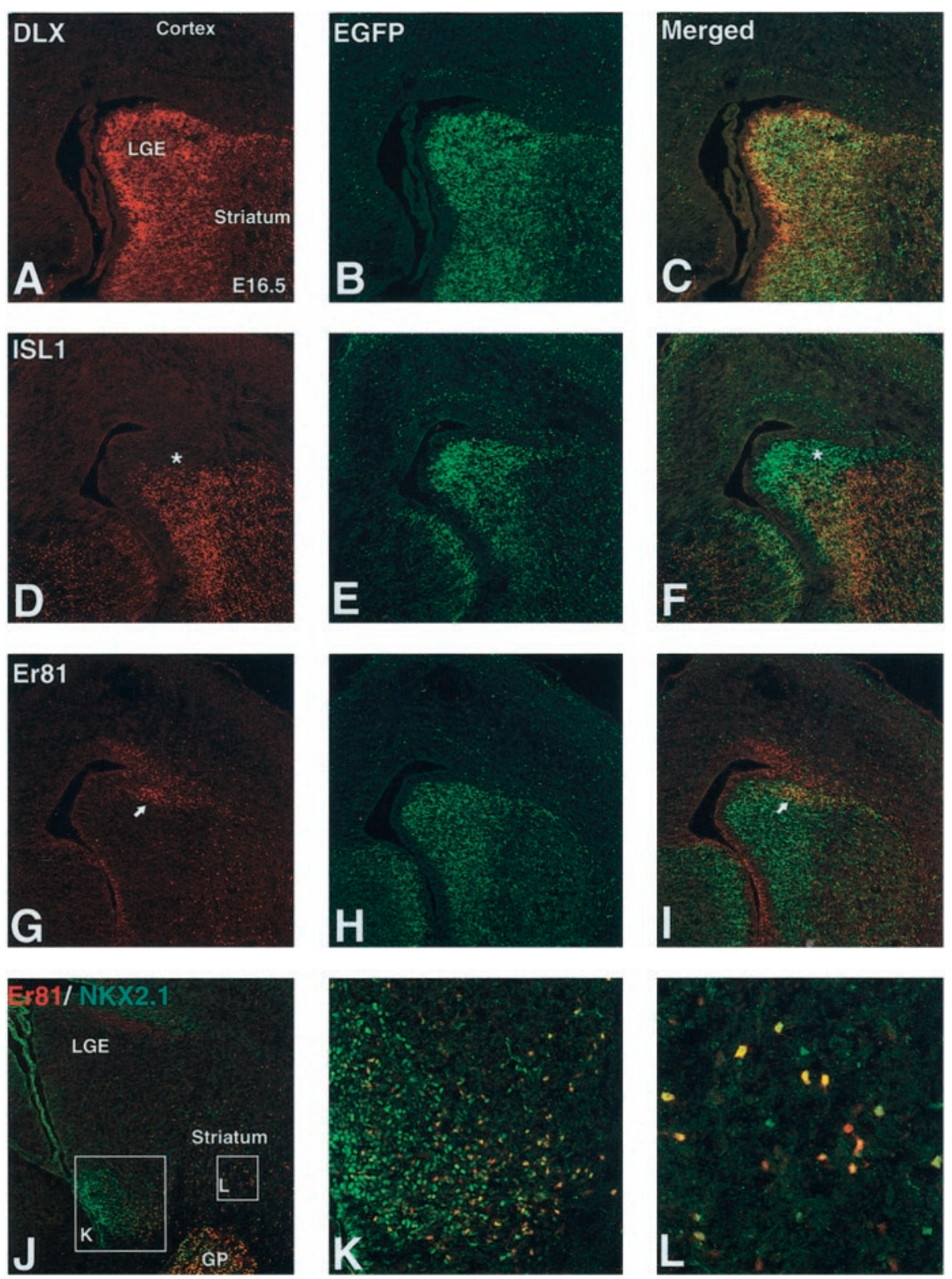

Figure2. Expression of DLX $(A), \operatorname{ISL1}(D)$, Er81 ( $G$ ), and EGFP expressed from the $D / x 5 / 6$ element $(B, E, H)$ atE16.5. Extensive overlap of $D L X(A)$ and EGFP ( $B$, C is seen in the SVZ of the LGE. As was the case atE12.5, the VZexpression of DLX Xroteins is notoverlapping with EGFP ( $(C$. ISL1 ( $D$ ) and the dorsal LGE contains Er87-expressing cells (arrow in G), which overlap with EGFP expression (arrowin/). Er81 is also found in scattered cells of the striatum and in the ventrolateral VZ of the pallium (G). Double-staining with Er81 (red) and NKX2.1 ( green) $(J-L)$ reveals coexpressing cells in the remnant of the MGE $(K)$ and in the globus pallidus (GP) as well as in scattered striatal neurons $(L)$.

single Gsh2 mutants (Toresson and Campbell, 2001); however, as in the Gsh2 mutants, ISL1 cells occupy the dorsal-most domain of the LGE (Fig. 6C). Er81 staining in the LGE SVZ shows a decreased number of cells, occupying a significantly smaller domain, in the Gsh2 (Fig. 6E) and Gsh1/2 mutants (Fig. 6F) compared with wild types (Fig. 6D). Hence, the $\mathrm{DLX}^{+} ; \mathrm{Er} 1^{+}$domain of the LGE SVZ appears to be severely depleted in Gsh mutants.

If our conjecture that the $\mathrm{DLX}^{+} ; \mathrm{Er} 81^{+}$domain of the LGE SVZ gives rise to olfactory bulb interneurons is correct, then there should be fewer Er81-positive neurons in the olfactory bulbs of Gsh2 and Gsh1/2 mutants. Indeed this seems to be the case. In Gsh2 mutants, only scattered cells expressing Er81 can be detected in the mantle of the olfactory bulb (Fig. $6 \mathrm{H}$ ), whereas the Gsh1/2 mutants show an even more severe reduction (Fig. 6I). 

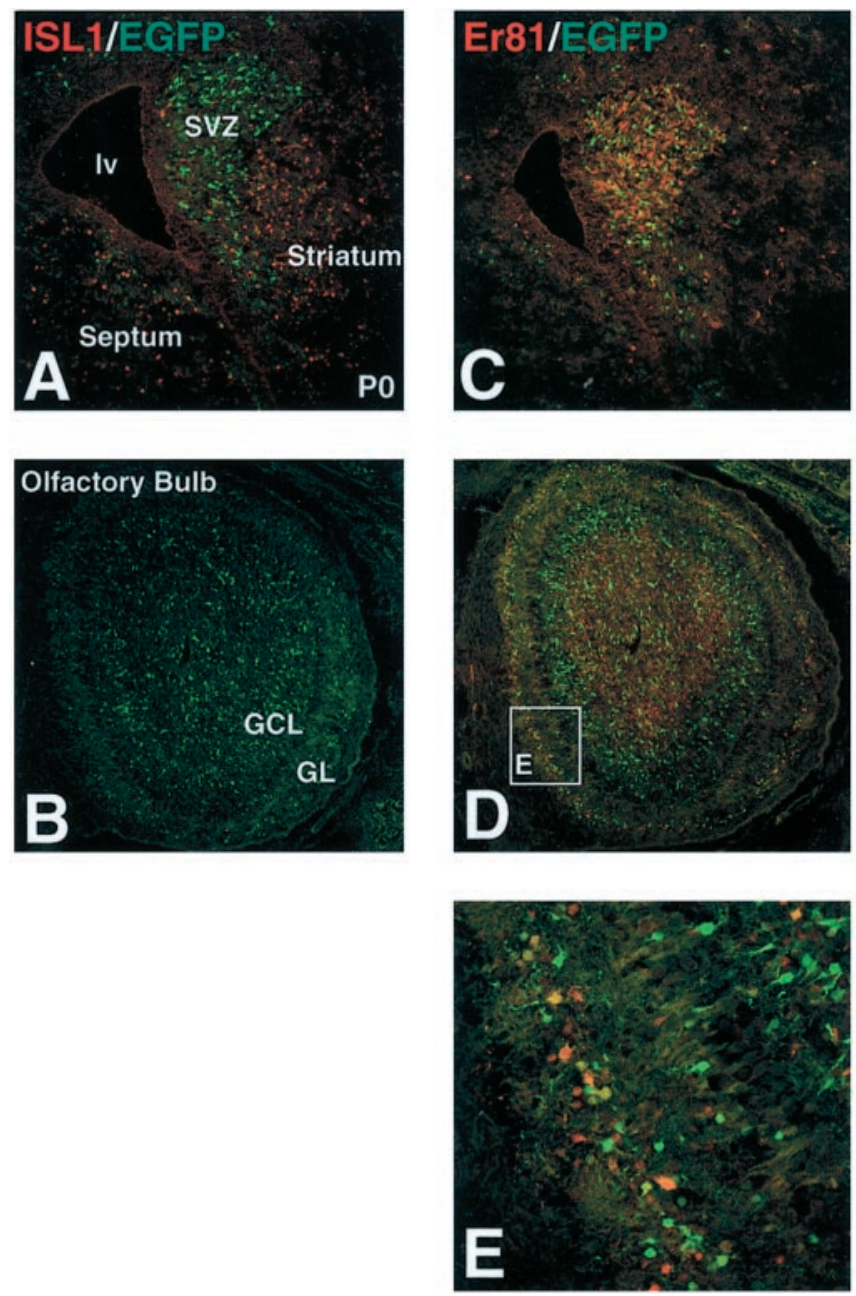

Figure 3. Expression of ISL1 $(A, B)$, Er81 ( $C-E)$, and EGFP expressed from the $D / x 5 / 6$ element $(A-E)$ at P0. At birth, few ISL1-positive cells remain in the EGFP-expressing SVZ region $(A)$. Furthermore, no ISL1 cells are detected in the newborn olfactory bulb, which contains many differentiating neurons positive for D1x5/6-driven EGFP $(B)$. Unlike ISL1, Er81 is found coexpressed with many EGFP cells of the SVZ ( $C$ ) as well as in many neurons of the olfactory bulb $(D$, $E$ ). E, High power of the outer mantle layer of the olfactory bulb (box in $D$ ) showing presumptive periglomerular neurons coexpressing Er81 and EGFP. GCL, Granule cell layer; GL, glomerular layer; $/ v$, lateral ventricle.

Despite the reduced staining in the mantle regions of the Gsh mutant olfactory bulbs, Er81 remains expressed in periventricular regions of the mutant olfactory bulb. As described above, at E12.5, a DLX ${ }^{-} ; \mathrm{Er} 81^{+}$domain is found at rostral levels in the primordia of the olfactory bulb. Thus it is possible that progenitors of the locally derived neurons (i.e., the projection neurons) also express this marker and downregulate it with differentiation.

Yun et al. (2001) have previously analyzed Er81 gene expression in Sey/Sey (i.e., Pax6) mutants. They showed that the expression of Er81 expands dorsally, similar to the other ventral regulators (e.g., Mash1, Gsh2, and Dlx genes). Our data are in agreement with this, however: because the Er81 protein expression is confined to the SVZ of the LGE, we observed an interesting change in its expression pattern within the developing cortex. In wild types at E14.5, Er81 is also expressed in the ventrolateral VZ of the pallium (Fig. 6J) (see also Fig. 2G, E16.5). Although the SVZ expression of Er81 is expanded dorsally in Sey/Sey mutants at this stage, it appears to be at the expense of its normal expression in the pallial VZ (Fig. $6 \mathrm{~K}$ ). However, this is in accordance with the dorsal shift of other ventral markers such as Gsh2,
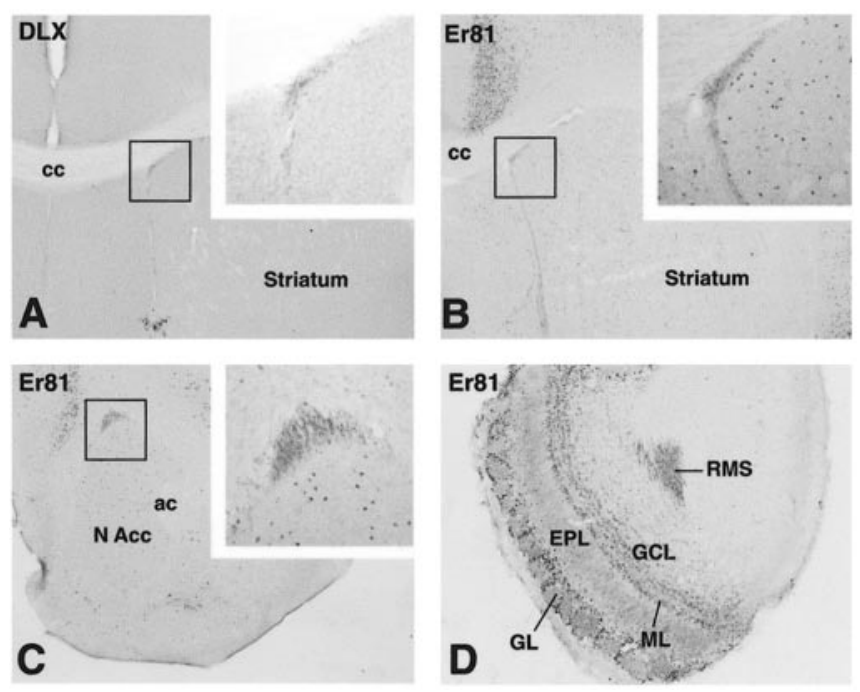

Figure 4. DLX and Er81 expression in the adult SVZ, RMS, and olfactory bulb. A, Although DLX proteins are not expressed in the adult striatum, many cells express DLX in the SVZ (high-power inset). B, Er81-positive cells are also found in the SVZ (high-power inset) as well as in the RMS ( $C$, $D)$. In the olfactory bulb, Er81 expression is observed in periglomerular cells and in granule cells of the mitral layer and outer portions of the granule cell layer. Note that Er81 is also observed in scattered striatal and nucleus accumbens neurons as well as in deep layers of the cerebral cortex. $a c$, Anterior commissure; $c c$, corpus callosum; EPL, external plexiform layer; $G C L$, granule cell layer; $G L$, glomerular layer; $M L$, mitral layer; $N$ Acc, nucleus accumbens.

Mash1, and Dlx genes (Stoykova et al., 2000, Toresson et al., 2000; Yun et al., 2001). Unfortunately, in Sey/Sey mutants the olfactory bulb does not form correctly (Anchan et al., 1997; Jimenez et al., 2000), so it is difficult to determine whether they would have altered numbers of interneurons.

\section{Discussion}

The results of this study demonstrate the existence of two distinct progenitor populations in the SVZ of the LGE. These progenitor pools subdivide the $D l x$-expressing region of the LGE into a $\mathrm{DLX}^{+} ; \mathrm{ISL}^{+}{ }^{+}$domain that occupies most of the LGE SVZ and a $\mathrm{DLX}^{+} ; \mathrm{Err1}^{+}$domain in the dorsal-most portion of the LGE. We propose that the $\mathrm{DLX}^{+} ; \mathrm{ISL}_{1}{ }^{+}$domain gives rise to striatal projection neurons, whereas the $\mathrm{DLX}^{+} ; \mathrm{Er} 81^{+}$domain contributes interneurons to the olfactory bulb.

\section{Early segregation of striatal projection neuron and olfactory} bulb interneuron precursors

Our results suggest that starting at approximately E12.5 in the mouse, spatially distinct pools of specified progenitors exist in the SVZ of the LGE that generate either striatal projection neurons or olfactory bulb interneurons. A number of facts support the notion that the $\mathrm{DLX}^{+} ; \mathrm{ISL}^{+}{ }^{+}$domain of the LGE SVZ gives rise to projection neurons of the striatum. First, many studies have shown that the LGE is the principal source for striatal projection neurons (Deacon et al., 1994; Olsson et al., 1995, 1997, 1998; Wichterle et al., 2001). Furthermore, the ISL1 expression in the LGE SVZ is continuous, with differentiating neurons in the perinatal striatum (Fig. 2D). ISL1 expression in the striatum is rapidly downregulated postnatally, so that the only striatal neurons that continue to express detectable levels of ISL1 are the cholinergic interneurons (Wang and Liu, 2001). This interneuronal subtype has been shown previously to derive from the adjacent MGE (Olsson et al., 1998; Marin et al., 2000). The DLX ${ }^{+}$;SL1 ${ }^{+}$ domain is likely to be heterogeneous, because the striatal projection neurons possess at least two distinct subtypes based on neu- 


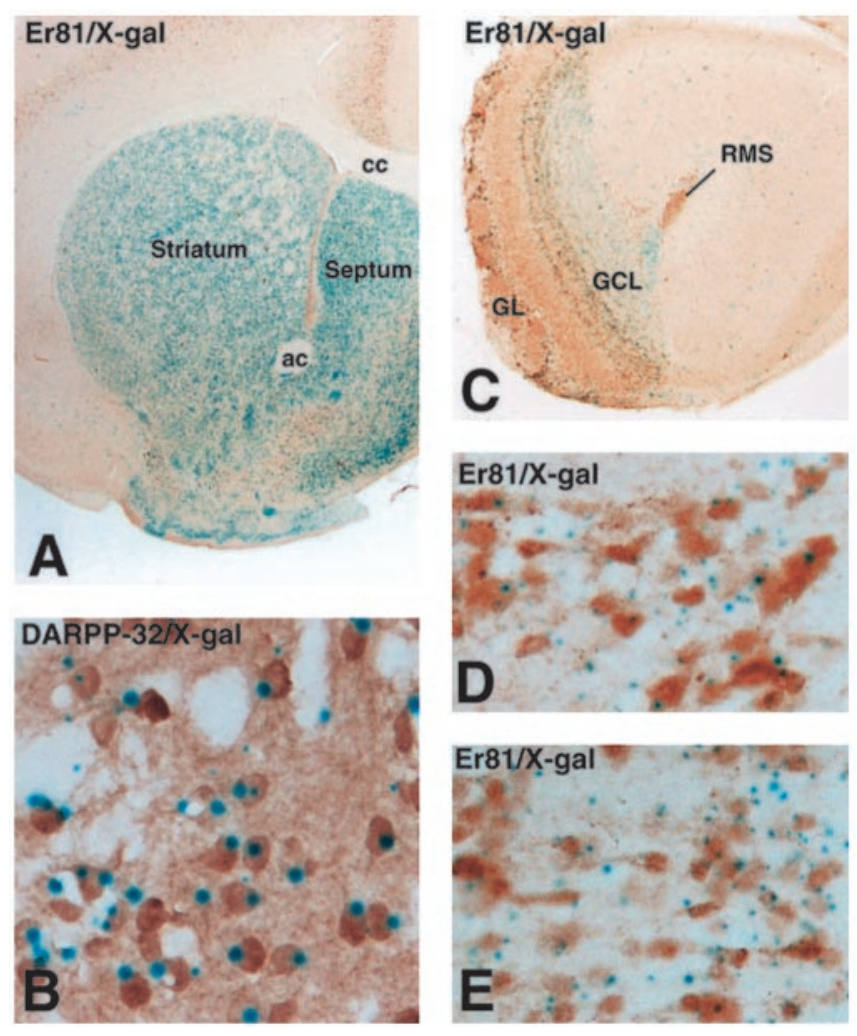

Figure 5. Fate mapping of $D / \times 5 / 6$-expressing cells. In thick sections (i.e., $30-40 \mu \mathrm{m}$ ) from mice double transgenic for D/x5/6-cre and gtROSA, many X-gal-positive cells (blue dots) are found in the adult striatum and septal regions $(A)$. In thin sections (i.e., $8 \mu \mathrm{m}$ ), most DARPP32-expressing cells in the striatum are found to be X-gal-positive $(B) . C, A$ stream of X-gal positive cells is seen exiting the RMS at olfactory bulb levels and labeling the granule cell layer $(\mathrm{GCL})$ and glomerular layer $(\mathrm{GL})$ in thick sections. In thin sections, nearly all Er81-positive periglomerular cells stain with X-gal (D), as was the case for Er81-expressing granule cells in the mitral layer (extreme left in $E$ ), and in the outer granule cell layers ( $E$ ). ac, Anterior commissure; cc, corpus callosum.

rochemical markers and efferent projections (for review, see Gerfen, 1992).

The present findings support the notion that the $\mathrm{DLX}^{+}$; Er81 ${ }^{+}$SVZ domain gives rise to olfactory bulb interneurons, including the periglomerular cells and at least a portion of the granule cells. Why do some granule cells display high levels of Er81 and others apparently none? It may be that all migrating and/or differentiating granule cells express Er81 and a subpopulation subsequently downregulate it after differentiation. It is possible, however, that the granule cell precursors are heterogeneous and a portion of them derive from an area distinct from the $\mathrm{DLX}^{+} ; \mathrm{Er} 81^{+}$domain. Presumably, this would also be in the $\mathrm{DLX}^{+}$;ISL1 ${ }^{-}$domain, because ISL1-positive cells are not seen in the RMS or olfactory bulb at embryonic or postnatal stages.

Despite their common expression of Er81, periglomerular and granule cells appear to derive primarily from separate progenitors. Using retroviral lineage methods, Reid et al. (1999) have shown that approximately one-half of the clones resulting from infection at early stages (i.e., E14-E15 in the rat; approximately E12-E13 in the mouse) contain multiple cell types (e.g., granule and periglomerular cells), whereas all of those infected at later stages (i.e., E17 in the rat; E15 in the mouse) contain only one cell type. Interestingly, no clones were reported to have dispersed between the developing striatum and olfactory bulb even after infection at early stages. The apparent lack of dispersion between the olfactory bulb and striatum could be attributable to a technical problem such as downregulation of the transgene in one of these regions (Halliday and Cepko, 1992). However, it could suggest that the separate populations of progenitors in the LGE SVZ described here arise from differently specified precursor cells in the VZ.

Although it has been well established that olfactory bulb interneurons migrate long distances rostrally to take up residence in the olfactory bulb at postnatal and adult stages (Luskin, 1993; Lois and Alvarez-Buylla, 1994; Lois et al., 1996; Wichterle et al., 1999), only recently has it become clear that this strategy is also used at embryonic stages. Previous studies have shown that significant numbers of periglomerular and granule cells are generated during the embryonic period (Hinds, 1968; Bayer, 1983). Using ultrasound-guided transplantation techniques, Wichterle et al. (2001) have demonstrated that genetically tagged LGE cells harvested from E13.5 embryos transplanted to a wild-type LGE of the same developmental stage migrate rostrally to the olfactory bulb as well as laterally to the adjacent striatum during embryogenesis. Interestingly, the authors noted that in embryos in which the donor LGE cells were injected into the anterior dorsal portion of the LGE, olfactory bulb interneurons in the glomerular and granule cell layers were consistently found. This correlates well with the position of the $\mathrm{DLX}^{+} ; \mathrm{Er} 81^{+}$domain described here.

Progenitors in the $\mathrm{DLX}^{+} ; \mathrm{Er} 81^{+}$domain of the LGE SVZ appear to be heavily dependent on the function of Pax6 and Gsh2. In the Gsh mutants, this domain is extinguished, whereas it expands dorsally in the Pax6 (Sey) mutants. At least in the Gsh mutants, the altered LGE expression of Er81 correlates with the loss of Er81-expressing interneurons in the mutant olfactory bulb. The lack of identifiable olfactory bulbs in Pax6 mutants (Anchan et al., 1997; Jimenez et al., 2000) unfortunately precludes additional analysis. Progenitors in the $\mathrm{DLX}^{+}{ }^{+} \mathrm{ISL}^{+}{ }^{+}$domain seem to be differentially affected in Pax6 and Gsh mutants. Indeed, in Pax6 mutants, the ISL1 expression domain does not appear to expand dorsally, whereas severe truncations of this domain are seen in Gsh2 and Gsh1/2 double mutants (Toresson et al., 2000; Toresson and Campbell, 2001).

The roles of ISL1 and Er81 in generating striatal projection neurons and olfactory bulb interneurons, respectively, are presently unclear. Whether they simply mark these populations or actively instruct their differentiation is an interesting question. Isl1 has been shown previously to be required for motor neuron development (Pfaff et al., 1996). Unfortunately, Isl1 homozygous mutants stop developing at approximately E10.5 and die soon after, which is before striatal neurogenesis is underway (Bayer and Altman, 1995). A targeted mutation in Er81 has been performed recently (Arber et al., 2000), and the mutants show defects in wiring between proprioceptive afferents and motor neurons in the spinal cord; however, no analysis of the olfactory bulb has yet been reported.

\section{Origins of the postnatal SVZ}

The fact that the same neuronal subtypes (i.e., granule and periglomerular cells) are generated in the olfactory bulb at both embryonic and postnatal time-points would suggest that the progenitors are similar and perhaps share a common origin in the LGE. Indeed cells of the postnatal and adult SVZ express many molecules typical of LGE precursors/progenitors, notably, DLX proteins and Er81. It is possible therefore that the $\mathrm{DLX}^{+}{ }_{;} \mathrm{Er}_{81}{ }^{+}$; ISL1 $^{-}$domain of the LGE SVZ is analogous to, and directly precedes, the formation of the postnatal/adult SVZ. However, we cannot exclude the possibility that some of Er81-expressing cells in the adult SVZ may derive from the $\mathrm{DLX}^{-} ; \mathrm{Er} 81^{+} \mathrm{VZ}$ domain of the ventrolateral pallium. Although the most prominent ap- 

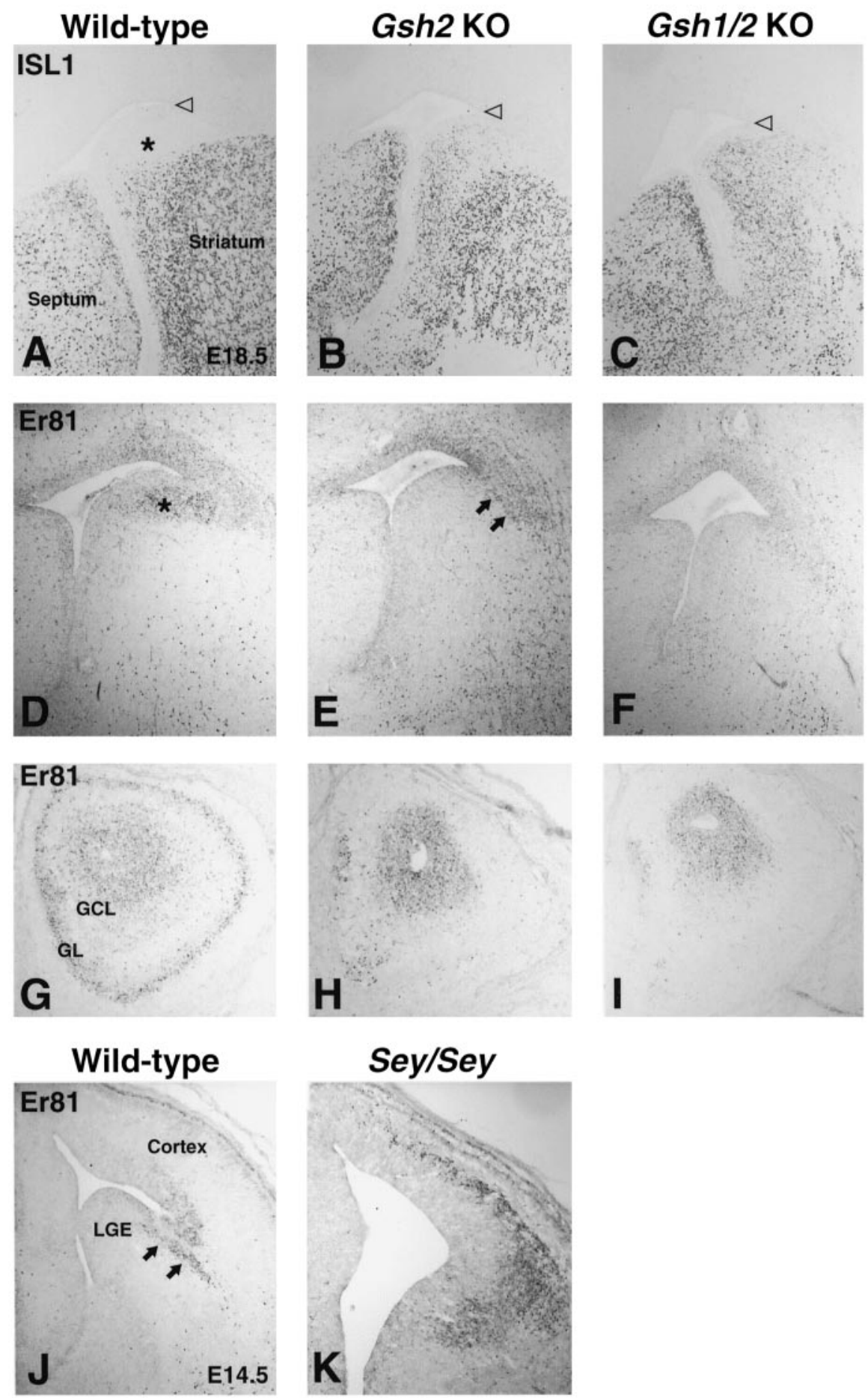

Figure 6. $A-I$, Altered expression of ISL1 and Er81 in Gsh and Sey homozygous mutants. ISL1 ( $A-C)$ and Er81 ( $D-l)$ expression in E18.5 wild-type $(A, D, G)$, Gsh2 $(B, E, H)$, and $G$ sh $1 / 2(C, F, I)$ mutants is shown. $A$, ISL1 expression is excluded from the dorsal-most portion of the wild-type LGE (asterisk). In Gsh2 (B) and GSh1/2 ( $C$ ) mutants, ISL1-positive cells are found in the dorsal LGE region. Open arrowheads in A-Cpoint to the LGE/cortex angle.D, Er81-positive cells are found in the dorsal (i.e., ISL1-negative; marked by an asterisk) LGE region of wild types. In Gsh2 (E) and Gsh1/2 ( $F$ ) mutants, few Er81-expressing cells are observed in the dorsal-most LGE (arrows in E). G, Er81-positive cells are found in periventricular regions as well is in the outer mantle regions of the olfactory bulb, presumably in the differentiating periglomerular cells. Although the periventricular Er81 staining remains in Gsh2 $(H)$ and Gsh1/2 (I) mutants, Er81-expressing cells in the olfactory bulb mantle are severely reduced in these mutants.J, K, Altered expression of Er81 expression in the Sey/Sey telencephalon. J, Er81-positive cells are present in the LGE SVZ (arrows) and in the VZ of the ventrolateral pallium of the E14.5 wild type. $K$, In Sey/Sey mutants at E14.5, pallial VZ expression is lost; however, SVZ expression expands into the dorsal telencephalon. GCL, Granule cell layer; GL, glomerular layer; KO, knockout. pearance of the postnatal/adult SVZ in coronal sections is in the dorsal-medial corner of the striatum, recent studies indicate that it actually exists as an intertwined network, with chains of migrating neurons covering most of the lateral ventricular wall and ultimately converging to form the RMS (Doetsch and AlvarezBuylla, 1996). It is easy to envision how the $\mathrm{DLX}^{+} ; \mathrm{Er} 81^{+}$domain could form the dorsal-medial portion of the SVZ, but the streams of SVZ cells at other positions on the ventricular wall would have to develop at postnatal stages. In fact, Er81 can be seen in clusters of SVZ cells at more ventral positions on the adult ventricular wall in coronal sections (data not shown). Moreover, whole-mount stains of the adult lateral ventricular wall show Er81positive cells scattered along the dorsoventral extent; however, obvious chains as demonstrated by TuJ1 (i.e., $\beta$-IIItubulin) staining (Doetsch and AlvarezBuylla, 1996) are not evident (our unpublished observations).

Considerable information exists as to the anatomical and ultrastructural components of the postnatal/adult SVZ. In this respect, the adult SVZ has been shown to be heterogeneous, with chains of migrating neuroblasts ensheathed by glial cells (Doetsch et al., 1997). The cellular components of the SVZ have been classified into groups based on their molecular and ultrastructural characteristics (Doetsch et al., 1997). The migrating neuroblasts have been termed " $A$ " cells, whereas the astroglial cells are called "B" cells. A third subtype, known as "C" cells, are present in clusters scattered throughout the SVZ. Only A and B cells are found in the RMS. Recently, the B cell (i.e., GFAP-positive, putative astrocyte), or a subpopulation thereof, has been suggested to represent the stem cell of the postnatal/adult SVZ (Doetsch et al., 1999). C cells are proposed to represent a transient amplifying population that gives rise to A cells (for review, see AlvarezBuylla and Garcia-Verdugo, 2002). It is currently unclear which subpopulation(s) of SVZ cells expresses Er81. However, it seems likely that the A cells (i.e., migrating neuroblasts) would express Er81 given its expression in the RMS and in differentiated neurons.

If the $\mathrm{DLX}^{+} ; \mathrm{Er} 1^{+}$domain of the LGE does correspond, at least in part, to the postnatal/adult SVZ, it provides an exciting possibility to identify this population of cells already at embryonic stages for more detailed studies. One important question is why these cells, or their pre- 
cursors, continue to undergo neurogenesis throughout the lifetime of the organism while their striatal counterparts (i.e., the $\mathrm{DLX}^{+}$; ISL1 ${ }^{+}$LGE domain) cease to generate neurons around birth.

\section{References}

Alvarez-Buylla A, Garcia-Verdugo JM (2002) Neurogenesis in adult subventricular zone. J Neurosci 22:629-634.

Anchan RM, Drake DP, Haines CF, Gerwe EA, LaMantia AS (1997) Disruption of local retinoid-mediated gene expression accompanies abnormal development in the mammalian olfactory pathway. J Comp Neurol 379:171-184.

Anderson KD, Reiner A (1991) Immunohistochemical localization of DARPP-32 in striatal projection neurons and striatal interneurons: implications for the localization of D1-like dopamine receptors on different types of striatal neurons. Brain Res 568:235-243.

Anderson SA, Qiu M, Bulfone A, Eisenstat DD, Meneses J, Pedersen R, Rubenstein JL (1997) Mutations of the homeobox genes Dlx-1 and Dlx-2 disrupt the striatal subventricular zone and differentiation of late born striatal neurons. Neuron 19:27-37.

Anderson SA, Marin O, Horn C, Jennings K, Rubenstein JL (2001) Distinct cortical migrations from the medial and lateral ganglionic eminences. Development 128:353-363.

Arber S, Ladle DR, Lin JH, Frank E, Jessell TM (2000) ETS gene Er81 controls the formation of functional connections between group Ia sensory afferents and motor neurons. Cell 101:485-498.

Bayer SA (1983) ${ }^{3} \mathrm{H}$-thymidine-radiographic studies of neurogenesis in the rat olfactory bulb. Exp Brain Res 50:329-340.

Bayer SA, Altman J (1995) Neurogenesis and neuronal migration. In: The rat nervous system (Paxinos G, ed), pp 1041-1078. San Diego: Academic.

Briscoe J, Pierani A, Jessell TM, Ericson J (2000) A homeodomain protein code specifies progenitor cell identity and neuronal fate in the ventral neural tube. Cell 101:435-445.

Bulfone A, Wang F, Hevner R, Anderson S, Cutforth T, Chen S, Meneses J, Pedersen R, Axel R, Rubenstein JL (1998) An olfactory sensory map develops in the absence of normal projection neurons or GABAergic interneurons. Neuron 21:1273-1282.

Casarosa S, Fode C, Guillemot F (1999) Mash1 regulates neurogenesis in the ventral telencephalon. Development 126:525-534.

Corbin JG, Gaiano N, Machold RP, Langston A, Fishell G (2000) The Gsh2 homeodomain gene controls multiple aspects of telencephalic development. Development 127:5007-5020.

Deacon TW, Pakzaban P, Isacson O (1994) The lateral ganglionic eminence is the origin of cells committed to striatal phenotypes: neural transplantation and developmental evidence. Brain Res 668:211-219.

Doetsch F, Alvarez-Buylla A (1996) Network of tangential pathways for neuronal migration in adult mammalian brain. Proc Natl Acad Sci USA 93:14895-14900.

Doetsch F, Garcia-Verdugo JM, Alvarez-Buylla A (1997) Cellular composition and three-dimensional organization of the subventricular germinal zone in the adult mammalian brain. J Neurosci 17:5046-5061.

Doetsch F, Caille I, Lim DA, Garcia-Verdugo JM, Alvarez-Buylla A (1999) Subventricular zone astrocytes are neural stem cells in the adult mammalian brain. Cell 97:703-716.

Gerfen CR (1992) The neostriatal mosaic: multiple levels of compartmental organization. Trends Neurosci 15:133-139.

Halliday AL, Cepko CL (1992) Generation and migration of cells in the developing striatum. Neuron 9:15-26.

Hinds JW (1968) Autoradiographic study of histogenesis in the mouse olfactory bulb. I. Time of origin of neurons and neuroglia. J Comp Neurol 134:287-304.

Jessell TM (2000) Neuronal specification in the spinal cord: inductive signals and transcriptional codes. Nat Rev Genet 1:20-29.

Jimenez D, Garcia C, de Castro F, Chedotal A, Sotelo C, de Carlos JA, Valverde F, Lopez-Mascaraque L (2000) Evidence for intrinsic development of olfactory structures in Pax-6 mutant mice. J Comp Neurol 428:511-526.

Lavdas AA, Grigoriou M, Pachnis V, Parnavelas JG (1999) The medial ganglionic eminence gives rise to a population of early neurons in the developing cerebral cortex. J Neurosci 19:7881-7888.

Li H, Zeitler PS, Valerius MT, Small K, Potter SS (1996) Gsh-1, an orphan Hox gene, is required for normal pituitary development. EMBO J 15:714-724.
Liu JK, Ghattas I, Liu S, Chen S, Rubenstein JL (1997) Dlx genes encode DNA-binding proteins that are expressed in an overlapping and sequential pattern during basal ganglia differentiation. Dev Dyn 210:498-512.

Lois C, Alvarez-Buylla A (1994) Long-distance neuronal migration in the adult mammalian brain. Science 264:1145-1148.

Lois C, Garcia-Verdugo JM, Alvarez-Buylla A (1996) Chain migration of neuronal precursors. Science 271:978-981.

Luskin MB (1993) Restricted proliferation and migration of postnatally generated neurons derived from the forebrain subventricular zone. Neuron 11:173-189.

Mao X, Fujiwara Y, Orkin SH (1999) Improved reporter strain for monitoring Cre recombinase-mediated DNA excisions in mice. Proc Natl Acad Sci USA 96:5037-5042.

Marin O, Rubenstein JL (2001) A long, remarkable journey: tangential migration in the telencephalon. Nat Rev Neurosci 2:780-790.

Marin O, Anderson SA, Rubenstein JL (2000) Origin and molecular specification of striatal interneurons. J Neurosci 20:6063-6076.

Olsson M, Campbell K, Wictorin K, Bjorklund A (1995) Projection neurons in fetal striatal transplants are predominantly derived from the lateral ganglionic eminence. Neuroscience 69:1169-1182.

Olsson M, Campbell K, Turnbull DH (1997) Specification of mouse telencephalic and mid-hindbrain progenitors following heterotopic ultrasound-guided embryonic transplantation. Neuron 19:761-772.

Olsson M, Bjorklund A, Campbell K (1998) Early specification of striatal projection neurons and interneuronal subtypes in the lateral and medial ganglionic eminence. Neuroscience 84:867-876.

Panganiban G, Sebring A, Nagy L, Carroll S (1995) The development of crustacean limbs and the evolution of arthropods. Science 270:1363-1366.

PfaffSL, Mendelsohn M, Stewart CL, Edlund T, Jessell TM (1996) Requirement for LIM homeobox gene Isl1 in motor neuron generation reveals a motor neuron-dependent step in interneuron differentiation. Cell 84:309-320.

Pleasure SJ, Anderson S, Hevner R, Bagri A, Marin O, Lowenstein DH, Rubenstein JL (2000) Cell migration from the ganglionic eminences is required for the development of hippocampal GABAergic interneurons. Neuron 28:727-740.

Reid CB, Liang I, Walsh CA (1999) Clonal mixing, clonal restriction, and specification of cell types in the developing rat olfactory bulb. J Comp Neurol 403:106-118.

Stoykova A, Treichel D, Hallonet M, Gruss P (2000) Pax6 modulates the dorsoventral patterning of the mammalian telencephalon. J Neurosci 20:8042-8050.

Stuhmer T, Puelles L, Ekker M, Rubenstein JL (2002) Expression from a Dlx gene enhancer marks adult mouse cortical GABAergic neurons. Cereb Cortex 12:75-85.

Sussell L, Marin O, Kimura S, Rubenstein JL (1999) Loss of Nkx2.1 homeobox gene function results in a ventral to dorsal molecular respecification within the basal telencephalon: evidence for a transformation of the pallidum into the striatum. Development 126:3359-3370.

Szucsik JC, Witte DP, Li H, Pixley SK, Small KM, Potter SS (1997) Altered forebrain and hindbrain development in mice mutant for the Gsh-2 homeobox gene. Dev Biol 191:230-242.

Toresson H, Campbell K (2001) A role for Gsh1 in the developing striatum and olfactory bulb of Gsh2 mutant mice. Development 128:4769-4780.

Toresson H, Potter SS, Campbell K (2000) Genetic control of dorsal-ventral identity in the telencephalon: opposing roles for Pax6 and Gsh2. Development 127:4361-4371.

Wang HF, Liu FC (2001) Developmental restriction of the LIM homeodomain transcription factor Islet-1 expression to cholinergic neurons in the rat striatum. Neuroscience 103:999-1016.

Wichterle H, Garcia-Verdugo JM, Herrera DG, Alvarez-Buylla A (1999) Young neurons from medial ganglionic eminence disperse in adult and embryonic brain. Nat Neurosci 2:461-466.

Wichterle H, Turnbull DH, Nery S, Fishell G, Alvarez-Buylla A (2001) In utero fate mapping reveals distinct migratory pathways and fates of neurons born in the mammalian basal forebrain. Development 128:3759-3771.

Yun K, Potter S, Rubenstein JL (2001) Gsh2 and Pax6 play complementary roles in dorsoventral patterning of the mammalian telencephalon. Development 128:193-205.

Zerucha T, Stuhmer T, Hatch G, Park BK, Long Q, Yu G, Gambarotta A, Schultz JR, Rubenstein JL, Ekker M (2000) A highly conserved enhancer in the Dlx5/Dlx6 intergenic region is the site of cross-regulatory interactions between Dlx genes in the embryonic forebrain. J Neurosci 20:709-721. 